\title{
Far upstream element-binding protein 1(FUBP1) expression differs between human colorectal cancer and non-cancerous tissue
}

\author{
M. Y. JIA, Y. J. WANG* \\ Department of Gastrointestinal, General Hospital of Jinan Military Command, Jinan 250031, China \\ ${ }^{*}$ Correspondence: yaojunwang@188.com
}

Received November 18, 2013 / Accepted January 30, 2014

\begin{abstract}
Presented study aimed to detect the expression of far upstream element-binding protein 1 (FUBP1) in clinical samples of colorectal carcinoma (CRC) and explore the correlations of their expression with the clinicopathological characteristics of CRC.

The streptavidin-perosidase (SP) method of immunohistochemistry was used to detect the expression of FUBP1 in 34 cases of colorectal cancer and their surrounding surrounding normal tissue, 30 cases of adenoma tissue. Using fluorescent quantitative reverse transcription polymerase chain reaction (qPCR), the expression of FUBP1 mRNA was measured in colorectal cancer and its surrounding normal tissue from 32 patients. FUBP1 protein expression level was detected by the Western blot method in 32 pairs of colorectal cancer tissue and surrounding normal tissue, and 30 cases of adenoma tissue.

The positive rate of FUBP1 was detected through histochemistry in colorectal carcinomas (82.3\%) which was higher than that in colorectal adenomas (46.7\%) and surrounding normal tissues (20.5\%). The relative amount of FUBP1 mRNA by qPCR method in colorectal carcinoma tissues $(0.2703 \pm 0.1118)$ was higher than that of surrounding normal tissues $(0.1898 \pm 0.0635$; $P<0.05)$. The Western blot showed that FUBP1 was mainly expressed in colorectal carcinoma tissues $(0.6499 \pm 0.1473)$, which barely expressed in adenoma tissues $(0.3756 \pm 0.1377 ; P<0.05)$ and surrounding normal tissues $(0.1675 \pm 0.0613 ; P<0.05)$.

FUBP1 expression differs among colorectal tissues, which is overexpressed in colorectal carcinoma tissue. Further studies are needed to explore the role of FUBP1 in the pathogenesis of colorectal carcinoma.
\end{abstract}

Key words: FUBP1, colorectal carcinoma, immunohistochemistry, qPCR, Western blot

The colorectal cancer (CRC) is one of the most common malignancies in the world [1]. Worldwidely, CRC is the $3 \mathrm{rd}$ of all malignant tumors and is the 4th leading cause of cancerrelated deaths. CRC is diagnosed in over one million persons annually [2]. As early as 1990, Fearon and Vogelstein pointed out that the development of colorectal cancer is a multistep process and that it involves the tumor-suppressor gene mutation deactivation and the accumulation of oncogene activation [3]. Over the past two decades, various inputs to cancer research have promoted the diagnosis and treatment of the colorectal cancer. The early colorectal cancer patients with 5 -year survival rate is about $90 \%$. However the overall survival of patients with advanced and metastatic still has not been obviously increased, which is only $15 \%$. As a result, many investigations have focused on the early diagnosis and prognosis of colorectal cancer, and a variety of biomarkers that could predict the survival of CRC has been reported recently [4-6].
It is well-known that c-myc is an important proto-oncogene and activation for c-myc will cause cells malignant transformation tendency. The far upstream element-binding protein 1 (FUBP1) plays an important role due to its transcriptional activity on the oncogene c-myc [7]. In the following years the studies found that the detection of a variety of tumor cell lines and tumor tissues including kidney cancer, bladder cancer, leukemia, ovarian cancer, breast cancer, osteosarcoma cells, cervical cancer showed these cells contained FUBPs family members and that the FUBP1 had high expression in the dividing cells, which is consistent with the c-myc expression [8-13]. To investigate the role of FUBP1 in the occurrence and development of CRC, we employed qPCR, Western blot and immunohistochemical methods to detect the expression of FUBP1 in clinical samples of CRC and then analyzed the correlations of their expression with the clinicopathological features of CRC. 


\section{Patients and methods}

Patients and tumor samples. From April 2011 to March 2012, fresh tissue samples were collected from 32 cases of CRC from the Department of Surgery, at General Hospital of Jinan Military Command of Chinese People's Liberation Army (PLA), and Affiliated Hospital of Taishan Medical University. The clinical samples were obtained from surgically removed and pathologically confirmed CRC. The patients included 18 men and 14 women and their mean age was 61.5 years old. None of the patients received preoperative radiotherapy or chemotherapy. The pathological classifications of CRC were based on sex, age, grade, tumor location, lymph node metastasis and distant metastasis. The matched surrounding normal tissue was obtained from a segment of the resected specimens that were the farthest from the tumor $(>5 \mathrm{~cm})$. Tissue samples were immediately snap-frozen in separate vials using liquid nitrogen and stored at $-80^{\circ} \mathrm{C}$ for the PCR and Western-blot. There were another 30 adenoma tissues removed from biopsy by colonoscopy for the Western-blot. The collection of clinical samples were approved by the Ethics Committee of General Hospital of Jinan Military Command of Chinese PLA and all patients gave their written informed consent. For immunohistochemistry we collected archived wax lumps of tissues from the pathology department of the same hospital from December 2011 to June 2012. They contained the colorectal cancer tissue 34 cases (19 men, 15 women; mean age, 60.87 years) and surrounding normal tissues in the colorectal cancer from tumor tissue $>1.5 \mathrm{~cm}$ which have none cancer confirmed by pathology, and 30 cases of adenoma tissues.

Immunohistochemistry. Two-micrometer-thick tissue sections were cut from the paraffin blocks containing tumor tissue, surrounding normal tissue and adenoma tissue, mounted on the object slide and subjected to immunohistochemistry using the standard streptavidin-peroxidase technique. The antibody used here was mouse anti-human FUBP1 monoclonal antibody (diluted 1:800; Santa Cruz Biotechnology Inc., Santa Cruz, CA, USA). The method followed the instructions of Sp - 9000 immunohistochemical staining kits and ZLI - 9033 concentrated DAB kit (ZSGB- Biotechnology Inc., Beijing, China). In each analysis, positive controls were used consisting of CRC samples previously shown to stain with the FUBP1 antibody. PBS in place of the primary antibody was used as a negative control.

Real-time quantitative PCR. We extracted the Total RNA from the tissues (50-100 mg) using Trizol (TaKaRa Inc., Japan) following the manufacturer's protocol. The cDNA synthesis was carried out by reverse transcription using a cDNA synthesis kit (PrimeScript ${ }^{\circledR}$ RT reagent Kit, TaKaRa Inc., Japan) following the manufacturer's protocol. PCR amplification of FUBP1 and GAPDH was done with SYBR Premix Ex Taq $^{\text {TM }}$ (TaKaRa Inc., Japan) with cDNA synthesized from the tissues. The primers used were as follows: FUBP1 (TaKaRa Inc., Japan): 5'-GGAACTCCAATGGGA CCATACAAC-3' (forward) and 5'-AGTGAGCGTAATAA GCAGCCCAAG-3' (reverse), amplicon 199 bp; GAPDH (Sangon Biotech(Shan ghai)Co.,Ltd) : 5'-GAAGGTGAAGGTCGGAGTC-3' (forward) and 5'-GAAGATGGTGATGGGATTTC-3' (reverse) amplicon 220 bp. Amplification conditions were as follows: Initial denaturation: $95^{\circ} \mathrm{C}$ for $5 \mathrm{~min}$, and the PCR proceeded for 40 cycles: $95^{\circ} \mathrm{C}$ for $5 \mathrm{~s}, 60^{\circ} \mathrm{C}$ for $20 \mathrm{~s}$. The expression level of FUBP1 was expressed as $2^{-\Delta \mathrm{Ct}}$, where $\Delta \mathrm{Ct}=\mathrm{Ct}(\mathrm{FUBP} 1)-\mathrm{Ct}$ (GAPDH). PCR products were visualized and photographed under ultraviolet light by $2 \%$ gel electrophoresis.

Western blot. Tissues were washed with ice-cold PBS prior to disruption on ice for ten minutes with Total Protein Lysis buffer supplemented with protease inhibitors (Thermo Fisher Scientific, CA, USA). Equal amounts of protein were separated by SDS-PAGE, transferred to Polyvinylidene Fluoride membranes (Thermo Fisher Scientific, CA, USA), which were activated by formaldehyde and then were blocked for 2 hours at $4^{\circ} \mathrm{C}$. The primary antibody, mouse anti-human FUBP1 monoclonal antibody (Santa Cruz Biotechnology Inc., Santa Cruz, CA, USA), was diluted 1:800 in 5\% BSA and incubated with the membrane overnight at $4^{\circ} \mathrm{C}$. The secondary antibody (Thermo Fisher Scientific, CA, USA) which was marked by horseradish peroxidase (HRP) was incubated for 4 hours at $4^{\circ} \mathrm{C}$ and visualised using electrochemiluminescence (ECL) developer. By using the ImageJ processing software (National Institutes of Health, USA) we analyzed the densitometric of band intensity.

Scoring and statistical analyses. Double-blind method was used to observe 5 horizons by two observers independently. Cores were scored for staining intensity of FUBP1-immunoreactive cells ( 0 , no staining; 1 , mild; 2 , moderate; 3 , strong) and percentage of the cells staining positively $(0,<5 \% ; 1,5-25 \% ; 2$, $26-50 \% ; 3,51-75 \% ; 4,>75 \%)$. These scores were multiplied to give final scores of $0-12$. A staining index of $<3$ was deemed to negative while $\geq 3$ was positive (3-5, weakly positive; $6-8$, moderate positive; 9-12, intense positive) [14]. Statistical analysis was performed by using SPSS17.0 software (SPSS, Inc., Chicago, IL, USA) and $P<0.05$ was considered significant. All the measurement data were expressed as mean $(\chi) \pm$ standard deviation(s). The differences between the CRC tissues and the surrounding normal tissues were analyzed using $\chi^{2}$, a paired samples $t$-test, or One - Way ANOVA (one - way Analysis of Variance), while categorical data were studied using the independent samples $t$-test or Rank-Sum test.

\section{Results}

Immunohistochemistry. The FUBP1 staining was detected in the nucleus. Immunohistochemical staining found that FUBP1 immunoreactivity was detected mainly in the nucleus of colorectal carcinoma cells but in the cytoplasm in the adjacent normal colorectal tissues (Fig. 1). The positive rates of FUBP1 in the tissues of colorectal carcinoma, surrounding normal tissues and adenoma tissues were $82.3 \%$ (28/34), 20.5\% (7/34), and 46.7\% (14/30), respectively. The difference in the positive rates of FUBP1 between the 

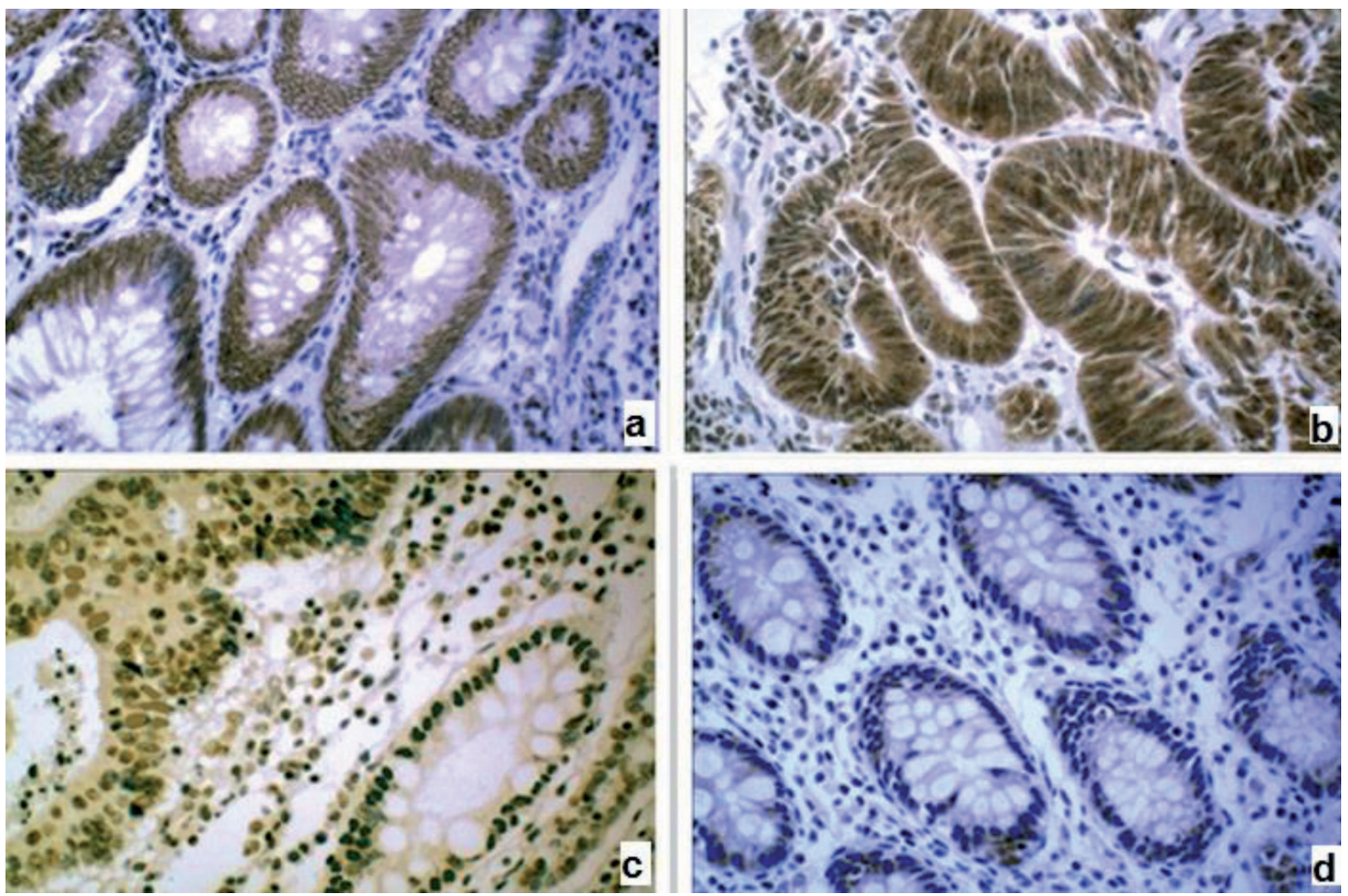

Figure 1. Immunohistochemical staining for FUBP1 in tissues of adenoma tissues, colorectal carcinoma tissues and surrounding normal tissues. a. FUBP1-positive staining in the nuclei in adenoma tissues; b. FUBP1-positive staining in the nuclei in colorectal carcinoma tissues; c. FUBP1-positive staining in the nuclei in colorectal carcinoma tissue and negative staining in surrounding normal tissues; d. FUBP1-negative staining in surrounding normal tissues.

colorectal carcinoma tissues and the other two tissues was statistically significant (all $P<0.05$ ) (Table 1 ). There were no significant correlations between FUBP1 expression and sex, age, grade, tumor location, lymph node metastasis or distant metastases (Table 2).

qPCR. We performed qPCR analysis to detect the expression of FUBP1 at mRNA level in 32 pairs of matched clinical samples. A melting curve analysis was performed after completion of the PCR (Fig. 2). The relative amount of FUBP1 mRNA with qPCR method in colorectal carcinoma tissues $(0.2703 \pm 0.1118)$ was higher than that of surrounding normal tissues $(0.1898 \pm 0.0635 ; P<0.05)$ (Table 3$)$. There were no significant correlations between FUBP1 expression and sex, age, grade, tumor location, lymph node metastasis or distant metastases (Table 4). Some samples of the electrophoresis assay results are shown in Fig. 3.

Table 1. FUBP1 expression in the colorectal carcinoma, Surrounding normal and adenoma tissue (cases, \%)

\begin{tabular}{lcccccc}
\hline Group & n & - & + & ++ & +++ & Rate (\%) \\
\hline colorectal carcinoma $^{(1)}$ & 34 & 6 & 9 & 11 & 8 & 82.3 \\
Surrounding ormalue $^{(2)}$ & 34 & 27 & 6 & 1 & 0 & 20.5 \\
adenoma tissue $^{(3)}$ & 30 & 16 & 11 & 2 & 1 & 46.7 \\
\hline
\end{tabular}

(1), ${ }^{2}: P=0.000 ;{ }^{(1)},{ }^{3}: P=0.003 ;{ }^{2},{ }^{(3)}: P=0.027$
Table 2. Correlation between FUBP1 expression and clinicopathological features of colorectal carcinoma

\begin{tabular}{|c|c|c|c|c|}
\hline \multirow{2}{*}{ Features } & \multirow{2}{*}{$\mathbf{n}$} & \multicolumn{2}{|c|}{ FUBP1 experssion } & \multirow{2}{*}{$P$ value } \\
\hline & & positive & Negtive & \\
\hline Sex & & & & 0.564 \\
\hline Male & 19 & 15 & 4 & \\
\hline Female & 15 & 13 & 2 & \\
\hline Age group (yr) & & & & 0.753 \\
\hline$<60$ & 15 & 12 & 3 & \\
\hline$>60$ & 19 & 16 & 3 & \\
\hline LN involvement & & & & 0.142 \\
\hline Yes & 15 & 14 & 1 & \\
\hline No & 19 & 14 & 5 & \\
\hline Distant metastasis & & & & 0.549 \\
\hline Yes & 9 & 8 & 1 & \\
\hline No & 25 & 20 & 5 & \\
\hline Dukes stage & & & & 0.634 \\
\hline $\mathrm{A}+\mathrm{B}$ & 20 & 17 & 3 & \\
\hline $\mathrm{C}+\mathrm{D}$ & 14 & 11 & 3 & \\
\hline Differentiation & & & & 0.111 \\
\hline High and medium & 25 & 19 & 6 & \\
\hline Low & 9 & 9 & 0 & \\
\hline Tumor location & & & & 0.231 \\
\hline Right colon & 13 & 12 & 1 & \\
\hline Left colon & 21 & 16 & 5 & \\
\hline
\end{tabular}

LN: lymph node 


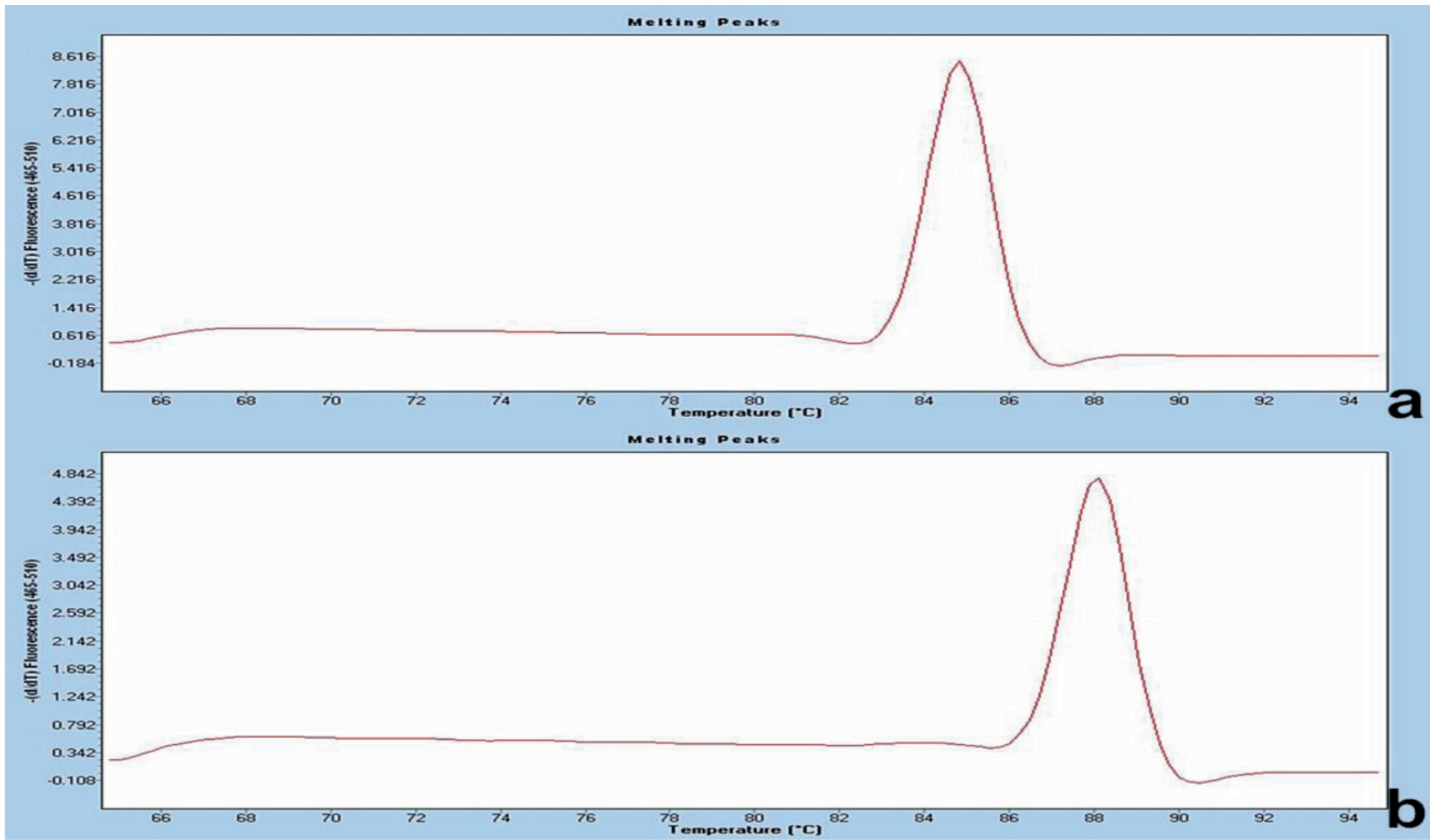

Figure 2. Typical amplification and melting curves of real-time RT-PCR for GAPDH (a) and FUBP1 (b). The sharp peak in each panel denotes the melting temperature of GAPDH $\left(84.83^{\circ} \mathrm{C}\right)$ and FUBP1 $\left(88.11^{\circ} \mathrm{C}\right)$.

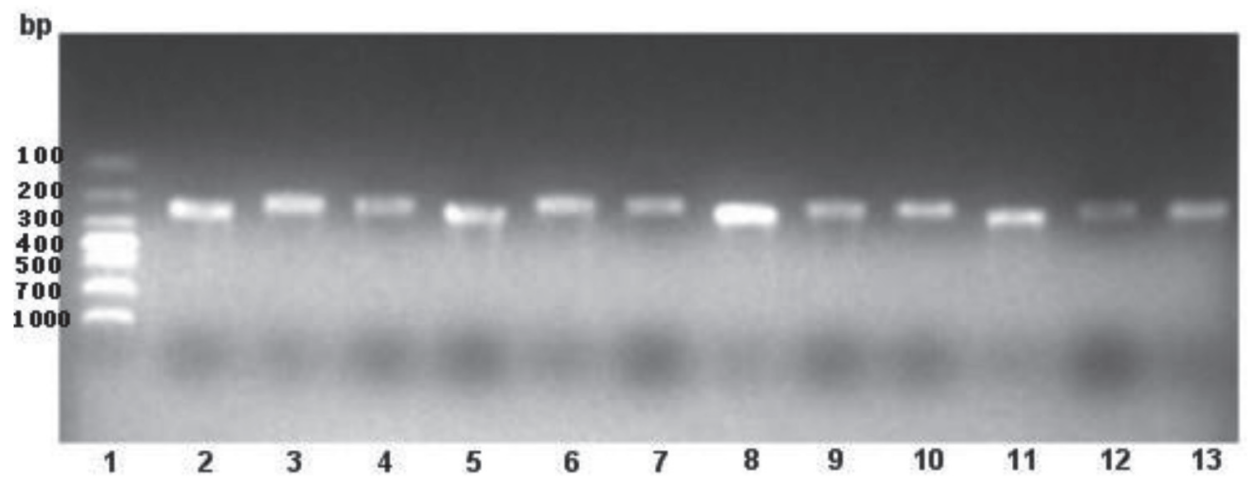

Figure 3. RT-PCR gel for GAPDH/FUBP1 mRNA in various colorectal tissues. (1). Marker; (2.5.8.11) RT-PCR products of GAPDH from colorectal carcinoma tissues; (3.6.9.12) RT-PCR products of FUBP1 from colorectal carcinoma tissues. (4.7.10.13); RT-PCR products of FUBP1 from surrounding normal tissues.

Table 3 Comparison of the relative amount of FUBP1 mRNA in colorectal carcinoma and surrounding normal tissue $($ mean $\pm \mathrm{SD})$

\begin{tabular}{lccccc}
\hline Group & $\mathbf{n}$ & Mean \pm SD & $t$ & $P$ & \multicolumn{2}{c}{ 95\% CI of the Difference } \\
\cline { 4 - 6 } & & & & & Lower \\
colorectal carcinoma & 32 & $0.2703 \pm 0.1118$ & & & 0.00149 \\
Surrounding normal tissue & 32 & $0.1898 \pm 0.0635$ & 2.179 & 0.046 & 0.13461 \\
\hline
\end{tabular}

CI: Confidence Interval 


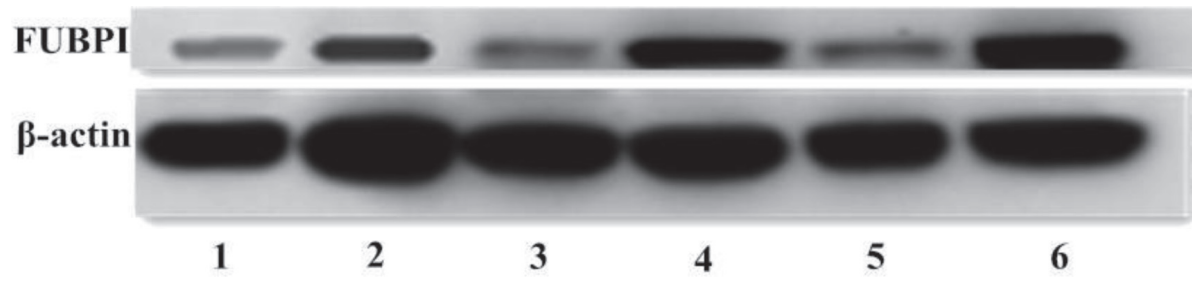

Figure 4. Western blot gel for $\beta$-actin/FUBP1 protein in various colorectal tissues. $(1,3,5)$ Western blot products from Surrounding normal tissues; $(2)$ Western blot products from colorectal carcinoma tissues; 4, 6) Western blot products from colorectal carcinoma tissues.

Western blot. FUBP1 protein expression level was detected by Western blot method in 32 pairs of colorectal cancer tissue and surrounding normal tissue, 30 cases of adenoma tissue. The FUBP1 was mainly expressed in colorectal carcinoma tissues $(0.6499 \pm 0.1473)$, barely expressed in surrounding normal tissues $(0.1675 \pm 0.0613 ; \mathrm{P}<0.05)$ and adenoma tissue $(0.3756 \pm 0.1377 ; P<0.05)$ (table 5$)$. Results of a typical experiment are shown in Fig. 4. Protein FUBP1 is found to be strongly expressed in tumor tissue and less in control tissue.

\section{Discussion}

FUBP1, a single-stranded DNA binding protein, is considered to be the ancestor of FUBPs family. Furthermore, being the most important transcription factor in FUBPs, FUBP1 has the functions of DNA helicase and RNA helicase [15], and can be combined with non-coding region of single-stranded far- upstream element (FUSE). Studies confirmed that many genes in cells contain FUSE sequences, in which the c-myc are the frequently researched and the most proto-oncogenes, whose activation will increase the malignant tendency of cells. FUBP1 play its regulatory function mainly by involving in FUSE / FUBP / FUBP Interacting Repressor (FIR) / Transcription Factor IIH (TFIIH) system. In this system, FUBP and FIR are a pair of functionally opposite and mutually collaborative proteins [16]. Through the FUSE identification in certain genes, like c-myc, FUBP activates the c-myc transcription and reaches the peak state by TFIIH stimulation. Meanwhile, under normal circumstances, FIR can be recruited by FUBP in combined FUSE / FUBP / TFIIH to play its control effect on the excessive transcription of c-myc so as to maintain the c-myc transcription to basic or relatively stable levels.

Numerous studies found that the detection of a variety of tumor cell lines and tumor tissues including kidney cancer, bladder cancer, leukemia, ovarian cancer, breast cancer, osteosarcoma cells, cervical cancer showed these cells contained FUBPS family members, and the FUBP1 had high expression in the dividing cells, which was consistent with the c-myc expression. The research on the digestive tract tumors showed that FUBP1 is an important Hepatic Cellular Cancer (HCC) tumor protein and induces tumor proliferation through direct or indirect inhibition of inhibitory factors and apoptosis genes in cell cycle [17-19]. In the earlier stage, we studied 30 primary
Table 4. Correlation between FBUP1 mRNA and clinicopathological features of colorectal carcinoma $($ mean $\pm \mathrm{SD})$

\begin{tabular}{lcccc}
\hline Features & $\mathbf{n}$ & Mean \pm SD & $t$ & $p$ \\
\hline Sex & & & 0.725 & 0.481 \\
Male & 18 & $0.2852 \pm 0.0943$ & & \\
Female & 14 & $0.2896 \pm 0.0839$ & & \\
Age group (yr) & & & -0.231 & 0.820 \\
$<60$ & 9 & $0.2522 \pm 0.0162$ & & \\
$>60$ & 23 & $0.2708 \pm 0.1775$ & & \\
LN involvement & & & -0.432 & 0.929 \\
Yes & 14 & $0.2844 \pm 0.0461$ & & \\
No & 18 & $0.2949 \pm 0.1163$ & & \\
Distant metastasis & & & -0.392 & 0.701 \\
Yes & 8 & $0.2848 \pm 0.1181$ & & \\
No & 24 & $0.2879 \pm 0.0855$ & & \\
Dukes stage & & & 0.323 & 0.771 \\
A+B & 19 & $0.2827 \pm 0.0639$ & & \\
C+D & 13 & $0.2946 \pm 0.1304$ & & \\
Differentiation & & & 0.638 & 0.534 \\
High and medium & 24 & $0.2995 \pm 0.1061$ & & \\
Low & 8 & $0.2666 \pm 0.0581$ & & \\
Tumor location & & & -0.693 & 0.545 \\
Right colon & 12 & $0.2514 \pm 0.0419$ & & \\
Left colon & 20 & $0.3086 \pm 0.1061$ & & \\
\hline
\end{tabular}

hepatocarcinoma tissues and surrounding normal tissues by immunohistochemistry and PCR. It has been found that the expression of both FUBP1 and c-myc is higher than that of surrounding normal tissues, and they are positively correlated [20]. FUBP1, as candidate indicators for early detection and diagnosis of colon cancer, can be combined with carcino-

Table 5. Comparison of the relative amount of FUBP1 protein in colorectal carcinoma and Surrounding normal tissue $($ mean \pm SD)

\begin{tabular}{lcccc}
\hline Group & $\mathbf{n}$ & Mean \pm SD & $\boldsymbol{F}$ & $\boldsymbol{P}$ \\
\hline colorectal carcinoma $^{(1)}$ & 32 & $0.6499 \pm 0.1473$ & 76.372 & 0.000 \\
Surrounding normal tissue $^{(2)}$ & 32 & $0.1675 \pm 0.0613$ & & \\
adenoma tissue $^{(3)}$ & 30 & $0.3756 \pm 0.1377$ & & \\
\hline (1). (2): $P=0.000$ : (1)、(3): $P=0.000$ : & (2), (3): $P=0.000$ & & &
\end{tabular}


embryonic antigen (CEA), CA199, CA72-4 or CA242 to be used as diagnostic and prognostic indicators, the physicians should take the CEA as the first choice [21]. Kuramitsu Y, et al [22] once used two-dimensional gel and mass spectrometry to compare the efficacy of gemcitabine (GEM) in treatment of sensitive pancreatic cancer cell lines (KLM1R) and gemcitabine-antagonistic pancreatic cancer cell lines (KLM1), and they found FUBP1 is down-regulated protein and that FUBP2 protein is up-regulated ones [22]. In this study, the immunohistochemistry, qPCR and Western blot were used to detect FUBP1 localization and expression intensity in colorectal cancer, adenoma and adjacent normal tissues, and we found: FUBP1 could be detected in various colorectal tissues, and its had different expression intensities in the cytoplasm and nucleus mRNA of different tissues. The cytoplasmic expression predominated for FUBP1 in adenoma and adjacent normal cells, while the nuclear expression predominated in colorectal cancer cells and the cytoplasmic expression was relatively weakened. The cytoplasmic, nuclear expressions in colorectal carcinoma tissues and adenoma, adjacent normal tissues of colorectal cancer are $82.3 \%$ (28/34), 20.5\% (7/34), and $46.7 \%$ (14/30),respectively. And the difference is statistically significant (all $P<0.05$ ). Meanwhile, the FUBP1 expression was found to be not significantly correlated with the age, sex, tumor location and degree of differentiation, lymph node metastasis and distant metastasis in studies about colorectal carcinoma tissues, which may be caused by small sample size or other influencing factors or unknown. This suggests that the abnormal expression of FUBP1 may occur during precancerous lesions and can be increasingly significant with the progression of the disease, which also plays a certain role in the occurrence and development of colorectal cancer, but this role is unobvious in invasion and metastasis. FUSE is a cis-acting element located on proto-oncogene c-myc and can be recognized by FUBP, while FUBP can be combined with FUSE, FIR, TFIIH to form a "molecular servo system" to regulate the c-myc gene transcription in a way of feedback. After p38, TGF- $\beta$ and other regulatory factors affect FUBP1 expression, the proto-oncogene c-myc transcription in this system is further affected, thus the slight changes of $\mathrm{c}$-myc is sufficient to cause the abnormal cell growths [23]. In addition, since the FUBP1 expression can affect the c-myc transcription, some studies show that it can achieve the effect of treating c-myc dependent tumor by influencing the FUBP1 expression. Huth et al. have confirmed that benzoylanthranilic acids can identify FUBP1 specifically and accurately. And benzoylanthranilic acids can combine with the DNA binding domain of FUBP1 and regulate FUBP1, thus inhibiting the c-myc activity [24]. Jang $M$ et al. have studied and found that when cells receive the apoptosis stimulation, due to the effect of cysteinyl aspartate specific proteinase, FUBP1 content in the cell nucleus is reduced, thus the contents of c-myc and a variety of targeted protein are decreased obviously. Therefore, after apoptosis induction, carcinogenic effect of c-myc is suppressed, as the result of FUBP1 fracture mediated by cysteinyl aspartate specific proteinase [25]. It is also found that the expression of FUBP1 is different in various colorectal tissue, which can further enrich the pathogenesis of colorectal cancer, and at the same time provide a new idea for diagnose and treatment of colorectal cancer.

\section{Conclusion}

FUBP1 expression differs among colorectal tissues, which is overexpressed in colorectal carcinoma tissue. Further studies are needed to explore the role of FUBP1 in the pathogenesis of colorectal carcinoma.

\section{References}

[1] JEMAL A, BRAY F, CENTER MM, FERLAY J, WARD E et al. Global cancer statistics. CA Cancer J Clin. 2011; 61: 69-90. http://dx.doi.org/10.3322/caac.20107

[2] CUNNINGHAM D, ATKIN W, LENZ HJ, LYNCH HT, MINSKY B et al. Colorectal cancer. Lancet. 2010; 375: 1030-47. http://dx.doi.org/10.1016/S0140-6736(10)60353-4

[3] FEARON ER, VOGELSTEIN B. A genetic model for colorectal tumorigenesis. Cell. 1990; 61: 759-67. http://dx.doi. org/10.1016/0092-8674(90)90186-I

[4] LI XD, MIAO SY, WANG GL, YANG L, SHU YQ et al. Amphiregulin and epiregulin expression in colorectal carcinoma and the correlation with clinicopathological characteristics. Onkologie. 2010; 33(7): 353-8. http://dx.doi.org/10.1159/ 000315380

[5] WANG F, ZHANG P, MA Y, YANG J, MOYER MP et al. NIRF is frequently upregulated in colorectal cancer and its oncogenicity can be suppressed by let-7a microRNA. Cancer Lett. 2012; 314: 223-31. http://dx.doi.org/10.1016/ j.canlet.2011.09.033

[6] XI RC, BIAO WS, GANG ZZ. Significant elevation of survivin and livin expression in human colorectal cancer: inverse correlation between expression and overall survival. Onkologie. 2011; 34(8-9): 428-32. http://dx.doi.org/10.1159/000331132

[7] ZHENG Y, MISKIMINS WK. Far upstream element binding protein 1 activates translation of p27Kip1 mRNA through its internal ribosomal entry site. Int J Biochem Cell Biol. 2011; 43: 1641-8. http://dx.doi.org/10.1016/j.biocel.2011.08.001

[8] BETTEGOWDA C, AGRAWAL N, JIAO Y, SAUSEN M, WOOD LD et al. Mutations in CIC and FUBP1 contribute to human oligodendroglioma. Science. 2011; 333: 1453-5. http://dx.doi.org/10.1126/science.1210557

[9] ENGIDAWORK E, AFJEHI-SADAT L, YANG JW, SLAVC I, LUBEC G. Protein chemical identification and characterization of the human variants of far upstream element binding protein in medulloblastoma DAOY cell line. Int J Oncol. 2006; 29: 721-36.

[10] GAGNE JP, GAGNE P, HUNTER JM, BONICALZI ME, LEMAY JF et al. Proteome profiling of human epithelial ovarian cancer cell line TOV-112D. Mol Cell Biochem. 2005; 275: 25-55. http://dx.doi.org/10.1007/s11010-005-7556-1

[11] SANZ R, ARAGUES R, STRESING V, MARTIN B, LANDEMAINE $T$ et al. Functional pathways shared by liver and lung metastases: a mitochondrial chaperone machine 
is up-regulated in soft-tissue breast cancer metastasis. Clin Exp Metastasis. 2007; 24: 673-83. http://dx.doi.org/10.1007/ s10585-007-9124-4

[12] SINGER S, MALZ M, HERPEL E, WARTH A, BISSINGER M et al. Coordinated expression of stathmin family members by far upstream sequence element-binding protein-1 increases motility in non-small cell lung cancer. Cancer Res. 2009; 69: 2234-43. http://dx.doi.org/10.1158/0008-5472.CAN-083338

[13] WEBER A, KRISTIANSEN I, JOHANNSEN M, OELRICH $\mathrm{B}, \mathrm{SCHOLMANN} \mathrm{K}$ et al. The FUSE binding proteins FBP1 and FBP3 are potential c-myc regulators in renal, but not in prostate and bladder cancer. BMC cancer. 2008; 8: 369. http://dx.doi.org/10.1186/1471-2407-8-369

[14] BRAUCKHOFF A, MALZ M, TSCHAHARGANEH D, MALEKN, WEBER A et al. Nuclear expression of the ubiquitin ligase seven in absentia homolog (SIAH)-1 induces proliferation and migration of liver cancer cells. J Hepatol. 2011; 55: 1049-57. http://dx.doi.org/10.1016/j.jhep.2011.02.019

[15] VINDIGNI A, OCHEM A, TRIOLO G, FALASCHI A. Identification of human DNA helicase $V$ with the far upstream element-binding protein. Nucleic Acids Res. 2001; 29: 1061-7. http://dx.doi.org/10.1093/nar/29.5.1061

[16] MICHELOTTI GA, MICHELOTTI EF, PULLNER A, DUNCAN RC, EICK D et al. Multiple single-stranded cis elements are associated with activated chromatin of the human c-myc gene in vivo. Mol Cell Biol. 1996; 16: 2656-69.

[17] CHEN M, ZHANG J, LI N, QIAN Z, ZHU M et al. Promoter hypermethylation mediated downregulation of FBP1 in human hepatocellular carcinoma and colon cancer. PLoS One. 2011; 6: e25564. http://dx.doi.org/10.1371/journal. pone.0025564

[18] RABENHORST U, BEINORAVICIUTE-KELLNER R, BREZNICEANU ML, JOOS S, DEVENS F et al. Overexpression of the far upstream element binding protein 1 in hepatocellular carcinoma is required for tumor growth.
Hepatology. 2009; 50: 1121-9. http://dx.doi.org/10.1002/ hep. 23098

[19] SANDERSON LM, BOEKSCHOTEN MV, DESVERGNE B, MULLER M, KERSTEN S. Transcriptional profiling reveals divergent roles of PPARalpha and PPARbeta/delta in regulation of gene expression in mouse liver. Physiol Genomics. 2010; 41: 42-52. http://dx.doi.org/10.1152/physiolgenomics. 00127.2009

[20] LU HN, ZHANG FC, WANG YJ. The effect of far upstream element binding protein-1 on the pathogenesis of human hepatocellular carcinomas. Chinese journal of hepatology. 2011; 19: 696-8.

[21] TACKE MD, BERNDT PC, HAGMANN M-LD, KARL JD, LANGEN HD et al., inventors; Use of the Far Upstream Element (FUSE) Binding Protein (FUBP) as a Marker for Colorectal Cancer2005.

[22] KURAMITSU Y, TABA K, RYOZAWA S, YOSHIDA K, ZHANG $\mathrm{X}$ et al. Identification of up- and down-regulated proteins in gemcitabine-resistant pancreatic cancer cells using two-dimensional gel electrophoresis and mass spectrometry. Anticancer Res. 2010; 30: 3367-72.

[23] KIM MJ, PARK BJ, KANG YS, KIM HJ, PARK JH et al. Downregulation of FUSE-binding protein and c-myc by tRNA synthetase cofactor p38 is required for lung cell differentiation. Nat Genet. 2003; 34: 330-6. http://dx.doi. org/10.1038/ng1182

[24] HUTH JR, YU L, COLLINS I, MACK J, MENDOZA R et al. NMR-driven discovery of benzoylanthranilic acid inhibitors of far upstream element binding protein binding to the human oncogene c-myc promoter. J Med Chem. 2004; 47: 4851-7. http://dx.doi.org/10.1021/jm0497803

[25] JANG M, PARK BC, KANG S, CHI SW, CHO S et al. Far upstream element-binding protein-1, a novel caspase substrate, acts as a cross-talker between apoptosis and the c-myc oncogene. Oncogene. 2009; 28: 1529-36. http://dx.doi. org/10.1038/onc.2009.11 